\title{
Micro-structured materials: inhomogeneities and imperfect interfaces in plane micropolar elasticity, a boundary element approach
}

Elena Atroshchenko ${ }^{1}$, Jack S. Hale ${ }^{2}$, Javier A. Videla ${ }^{1}$, Stanislav Potapenko ${ }^{3}$, and Stéphane P.A. Bordas $2,4,5$

\footnotetext{
${ }^{1}$ Department of Mechanical Engineering, University of Chile, Santiago, 8370448, Chile ${ }^{2}$ Institute for Computational Engineering, Faculté des Sciences, de la Technologie et de la Communication 6, University of Luxembourg, Luxembourg

${ }^{3}$ Department of Civil and Environmental Engineering, University of Waterloo, ON, Canada

${ }^{4}$ Cardiff University, Institute of Mechanics and Advanced Materials, School of Engineering, College of Physical Sciences and Engineering, UK.

${ }^{5}$ University of Western Australia, Intelligent Systems for Medicine Laboratory, School of Mechanical and Chemical Engineering, Australia.
}

October 28, 2016 
Abstract. In this paper we tackle the simulation of microstructured materials modelled as heterogeneous Cosserat media with both perfect and imperfect interfaces. We formulate a boundary value problem for an inclusion of one plane strain micropolar phase into another micropolar phase and reduce the problem to a system of boundary integral equations, which is subsequently solved by the boundary element method. The inclusion interface condition is assumed to be imperfect, which permits jumps in both displacements/microrotations and tractions/couple tractions, as well as a linear dependence of jumps in displacements/microrotations on continuous across the interface tractions/couple traction (model known in elasticity as homogeneously imperfect interface). These features can be directly incorporated into the boundary element formulation.

The BEM-results for a circular inclusion in an infinite plate are shown to be in an excellent agreement with the analytical solutions. The BEM-results for inclusions in finite plates are compared with the FEM-results obtained with FEniCS.

Keywords. Cosserat elasticity, boundary integral equation method, inclusion, FEniCS.

\section{Introduction}

This paper presents the first application and verification of the boundary element method to simulate the mechanical effects of inclusions with imperfect interfaces in plane micropolar elasticity.

Modern nano-technological applications such as sensors and actuators, microelecromechanical systems, electronic packaging, advanced nano-composites call for efficient approaches to model the mechanical behaviour of micro and nano-structured materials. Atomistic simulations are one way forward, but these are extremely computationally expensive $\varrho^{1}$, such that multi-scale approaches are required e.g. see Talebi et al. [2014]. One approach to account for the multi-scale nature of materials is to build continuum scale constitutive theories able to reproduce the continuum behaviour of such nano/micro-structured materials, see e.g. Mühlhaus et al. 1995] for an account of continuum models of micro-structured materials. The micropolar theory is one such approach, which we use in this paper.

Micropolar (also known as Cosserat) elasticity was first introduced by the Cosserat brothers Cosserat and Cosserat [1896 and further developed by Eringen 1965], Nowacki [1986], Eremeyev et al. [2013] etc., and it is able to account for the rotation of individual material points (differential elements). This leads to the description of a deformed state in terms of asymmetric stress and couple stress tensors. It was shown that micropolar constitutive models, in spite of being a continuum model, are able to replicate the experimentally-observed behavior of natural or engineered materials possessing micro or nano structures Mühlhaus et al. [1995] such as bone Yang and Lakes [1982], Park and Lakes [1986], Fatemi et al., Ramzani et al. [2012], fibre-reinforced composites Bigoni and Drugan [2006], Chen et al. [2009], Beveridge et al. [2013], blocky and layered materials, such as rock and rock masses Mühlhaus [1993], Adhikary and Dyskin 1997], Riahi and Curran [2010], cellular materials Onck [2002], Tekoglu and Onck and many others.

The problem of (imperfect) interfaces (also known as interphases) in Cosserat matter was scarcely addressed Duan et al. [2005], whilst it was much more intensively modelled and simulated in the context of standard linear elasticity, with or without surface effects, see e.g. Paggi and Wriggers 2012, Zhao et al. 2013a. b] and Nairn 2007, Yvonnet et al. $2008 \mathrm{~b}$ a for implementation aspects. It is however interesting to note that Cosserat mate-

\footnotetext{
${ }^{1}$ some estimates claim that it will be 80 years before the failure of one cubic centimeter of metal can be simulated using such approaches Al-Rub 2013.
} 
rials have been themselves used to model the mechanical effects of such interphases within heterogeneous materials, as discussed in depth in recent literature Dong et al. [2014, 2015].

Due to the rapid development of composite materials for advanced engineering applications, the problem of quantifying the effects of heterogeneities is crucially important, in particular in cases where the interfaces between the bulk/matrix and the inclusions are imperfect or carry surface energy.

The effects of heterogeneities/inhomogeneities have been studied well within the confines of Cauchy continua (classical elasticity), both analytically and numerically, starting from the classical Muskhelishvili's problem of a circular inclusion in an infinite plate Muskhelishvili [1977] to the finite and boundary element analysis of multiple inclusions of various shapes, see for example Mogilevskaya and Crouch [2002], Liu et al. [2005], Huang et al. [2014] and crack/inclusion interactions, e.g. Xue-Hui and Erdogan [1986 and more recently Natarajan et al. [2014].

In Cosserat elasticity, however, less work has been done and much remains to be understood about Cosserat-heterogeneous materials. Such efforts date back to 1976 with the work of Gupta 1976]. In the 1990s significant work has been done on Cosserat-heterogeneous materials to study the effects of inclusions Jasiuk and Ostoja-Starzewski 1995 and compute homogenized properties and their bounds and to understand their asymptotic behaviour Dendievel et al. [1998], Forest et al. [1999, 2001]. An interesting result of Forest et al. [2001] is that if $\ell$ is the size of the Cosserat-heterogeneities, $\ell_{c}$ the Cosserat intrinsic length scales and $L$ the size of the material sample, $\ell \approx \ell_{c} \ll L$ leads to a Cauchy continuum, whereas if $\ell_{c} \approx L$ then, the effective (homogenized) medium is better approximated by a Cosserat material.

More recently, work on Cosserat-heterogeneous materials has intensified somewhat with the work of Lubarda [2003], who provides analytical solutions in plane strain and Dong et al. [2014, 2015] who focus on the modelling of interphases in heterogeneous materials by a non-linear Cosserat material.

A number of analytical and numerical methods have been developed to treat boundary value problems of micropolar elasticity. The finite element method remains the most common tool of numerical analysis Li and Xie [2004], Jeong et al. [2009], Bauer et al. [2010], Natarajan et al. [2012], Natarajan [2014].

Recently, the boundary element method Atroshchenko and Bordas [2015], Hadjesfandiari and Dargush [2012] has been emerging as a powerful alternative due to its advantage in treating problems with non-smooth boundaries and infinite domains. For example, in Atroshchenko and Bordas 2015 the dual boundary element method was applied to crack problems in plane strain micropolar continua.

One of the advantages of using boundary elements for inclusion problems, is the ability to incorporate the model of imperfect interfaces directly into the boundary integral formulation, keeping the linear formulation of the problem, while in the case of finite element method such interface model would make the formulation nonlinear. In this work we use the simple imperfect interface model, known as homogeneously imperfect interface, which is characterised by tractions and couple traction being continuous across the interface, and proportional to the jumps in displacements and out-of-plane microrotation. This model, for a circular inclusion in a plate subjected to uni-axial tension was investigated analytically in Videla and Atroshchenko with the full solution available in Atroshchenko [b].

Another imperfect interface model, used in this work, is characterized by arbitrary jumps in both surface tractions/couple traction, as well as in displacements and out-ofplane microration. Physically, such a model allows to impose more general boundary conditions, while mathematically it brings additional advantages for the problems in infinite 
domain, because it enables to significantly reduce the size of the problem by transferring the boundary conditions at infinity to the boundary conditions on the inclusion interface.

In this paper we develop a system of boundary integral equations for an inclusion problem in plane micropolar and solve it by the boundary element method. We show the excellent agreement of the BEM-results with the analytical and FEM-solutions. We present the BEM-study of micropolar effects on inclusions of various shapes in various loading conditions. We demonstrate the dependence of the stress concentration factors on material parameters, including the limiting cases, when one material is nearly classical, while the second one is strongly micropolar.

The paper is organized as follows. In chapter 2 we formulate the boundary value problem of an inclusion in micropolar plane strain. In chapter 3 we derive the system of boundary integral equations. In chapter 4 we briefly outline the boundary element method procedure. Numerical results are given in chapter 5 , while chapter 6 contains discussion of the results and directions of future work.

\section{Mathematical formulation of an inclusion problem.}

According to Eringen [1965], a plane strain deformation of a micropolar material is described by two in-plane displacements $u_{1}=u_{1}(\boldsymbol{x}), u_{2}=u_{2}(\boldsymbol{x})$ and one out-of-plane microrotation $\phi_{3}=\phi_{3}(\boldsymbol{x})$, where $\boldsymbol{x}=\left(x_{1}, x_{2}\right)$, which we combine into one vector of generalized displacements: $\boldsymbol{u}=\left(u_{1}, u_{2}, u_{3}\right)^{T}$ with $u_{3}=\phi_{3}$. In absence of body forces and couples, the equations of equilibrium for a material described by parameters $\lambda, \mu, \kappa$ and $\gamma$ can be written as

$$
L\left(\partial_{x}\right) \boldsymbol{u}=0,
$$

where the matrix differential operator $L\left(\partial_{x}\right)=L\left(\xi_{\alpha}\right)$ is given in Schiavone, Iean [1970] as

$$
L\left(\xi_{\alpha}\right)=\left(\begin{array}{ccc}
(\lambda+\mu) \xi_{1}^{2}+(\mu+\kappa) \Delta & (\lambda+\mu) \xi_{1} \xi_{2} & \kappa \xi_{2} \\
(\lambda+\mu) \xi_{1} \xi_{2} & (\lambda+\mu) \xi_{2}^{2}+(\mu+\kappa) \Delta & -\kappa \xi_{1} \\
-\kappa \xi_{2} & \kappa \xi_{1} & \gamma \Delta-2 \kappa
\end{array}\right)
$$

with $\xi_{\alpha}=\partial / \partial x_{\alpha}$ and $\Delta=\partial^{2} / \partial x_{1}^{2}+\partial^{2} / \partial x_{2}^{2}=\xi_{1}^{2}+\xi_{2}^{2}$.

Two tractions $t_{1}=t_{1}(\boldsymbol{x}), t_{2}=t_{2}(\boldsymbol{x})$ and one couple-traction $t_{3}=t_{3}(\boldsymbol{x})$, defined on a boundary with normal $\boldsymbol{n}=\left(n_{1}, n_{2}\right)^{T}$, are also combined into vector $\boldsymbol{t}=\left(t_{1}, t_{2}, t_{3}\right)^{T}$. By the standard definition

$$
t_{\alpha}=\sigma_{\beta \alpha} n_{\beta}, \quad t_{3}=m_{\beta 3} n_{\beta}, \quad \alpha, \beta=1,2 .
$$

where $\sigma_{11}, \sigma_{12}, \sigma_{21}, \sigma_{22}$ are components of the asymetric micropolar stress tensor and $m_{13}$, $m_{23}$ are the couple-stresses.

Together with $L\left(\xi_{\alpha}\right)$ the boundary stress operator $T\left(\partial_{x}\right)=T\left(\xi_{\alpha}\right)$ is considered (Schiavone), which is defined by the following equation:

$$
\begin{aligned}
& T\left(\xi_{\alpha}\right)= \\
& \left(\begin{array}{ccc}
(\lambda+2 \mu+\kappa) \xi_{1} n_{1}+(\kappa+\mu) \xi_{2} n_{2} & \lambda \xi_{2} n_{1}+\mu \xi_{1} n_{2} & \kappa n_{2} \\
\mu \xi_{2} n_{1}+\lambda \xi_{1} n_{2} & (\mu+\kappa) \xi_{1} n_{1}+(\lambda+2 \mu+\kappa) \xi_{2} n_{2} & -\kappa n_{1} \\
0 & 0 & \gamma \xi_{\alpha} n_{\alpha}
\end{array}\right)
\end{aligned}
$$

Operator $T\left(\partial_{x}\right)$ is defined according to the stress strain relations and the constitutive equations, as given in Eringen 1965 in such a way that

$$
\boldsymbol{t}=T\left(\partial_{x}\right) \boldsymbol{u} .
$$


Together with constants $\lambda, \mu, \gamma, \kappa$, we use engineering constants: $G$ (shear modulus), $\nu$ (Poisson's ratio), $\ell$ (characteristic length) and $N$ (coupling number), defined in Yang and Lakes 1982.

We consider a bounded inclusion occupying the domain $S^{i}$ with the boundary $\partial S^{i}$ and inner normal $\vec{n}$ as shown in Fig.1. The inclusion is made of homogeneous and isotropic micropolar material with elastic constants $\lambda^{i}, \mu^{i}, \kappa^{i}, \gamma^{i}$. The matrix, which occupies domain $S^{e}$ is also homogeneous and isotropic micropolar material with elastic constants $\lambda^{e}, \mu^{e}, \kappa^{e}$, $\gamma^{e}$. The engineering material parameters, describing the inclusion or the matrix are denoted as $G^{i}, \nu^{i}, \ell^{i}, N^{i}$ or $G^{e}, \nu^{e}, \ell^{e}, N^{e}$ respectively.

Let $L^{i}\left(\partial_{x}\right)$ and $L^{e}\left(\partial_{x}\right)$ be the operator $L\left(\partial_{x}\right)$ with constants $\lambda^{i}, \mu^{i}, \kappa^{i}, \gamma^{i}$ and $\lambda^{e}, \mu^{e}, \kappa^{e}$, $\gamma^{e}$ respectively. The boundary stress operators $T^{i}\left(\partial_{x}\right)$ and $T^{e}\left(\partial_{x}\right)$ are defined analogously. The displacement vector in domain $S^{i}$ is denoted as $\boldsymbol{u}^{i}$, in domain $S^{e}$ as $\boldsymbol{u}^{e}$. The boundary tractions are defined as

$$
\boldsymbol{t}^{i}=T^{i}\left(\partial_{x}\right) \boldsymbol{u}^{i}, \boldsymbol{t}^{e}=T^{e}\left(\partial_{x}\right) \boldsymbol{u}^{e} .
$$

The first boundary value problem for an inclusion with the imperfect interface, that we consider in this paper, is given as follows:

$$
\begin{aligned}
L^{i}\left(\partial_{x}\right) \boldsymbol{u}^{i} & =0 \text { in } S^{i}, \\
L^{e}\left(\partial_{x}\right) \boldsymbol{u}^{e} & =0 \text { in } S^{e}, \\
\boldsymbol{u}^{e} & =\tilde{\boldsymbol{u}}^{e} \text { on } \partial S_{u}^{e}, \\
\boldsymbol{t}^{e} & =\tilde{\boldsymbol{t}}^{e} \text { on } \partial S_{t}^{e}, \\
\boldsymbol{u}^{e}-\boldsymbol{u}^{i} & =\boldsymbol{f} \text { on } \partial S^{i}, \\
\boldsymbol{t}^{e}-\boldsymbol{t}^{i} & =\boldsymbol{g} \text { on } \partial S^{i},
\end{aligned}
$$

where $\tilde{\boldsymbol{u}}$ is the generalized displacement vector, consisting of two displacements and one microrotation prescribed on Dirichlet part $\partial S_{u}^{e}$ of the outer boundary $\partial S^{e}$ and vector $\tilde{\boldsymbol{t}}$ is the generalized traction vector, consisting of two tractions and one couple traction prescribed on Neumann part $\partial S_{t}^{e}$ of $\partial S^{e}$. Jump in displacements and micro-rotation along the inclusion interface is described by function $\boldsymbol{f}=\left(f_{1}, f_{2}, f_{3}\right)^{T}$, while jump in tractions and couple tractions is given by $\boldsymbol{g}=\left(g_{1}, g_{2}, g_{3}\right)^{T}$. A perfect interface is characterized by $\boldsymbol{f}=\{0,0,0\}$ and $\boldsymbol{g}=\{0,0,0\}$.

Together with the interface boundary conditions given by the last two equations of (7) we consider the case of so-called homogeneously imperfect interface characterized by continuous stresses and jumps in the normal and tangential displacements proportional to the corresponding stress components. In micropolar elasticity two additional conditions need to be imposed, namely, continuous couple traction and jump in the microrotations proportional to the couple traction Videla and Atroshchenko. These conditions are written as:

$$
\begin{aligned}
\sigma_{n n}^{e} & =\sigma_{n n}^{i}, \\
\sigma_{n t}^{e} & =\sigma_{n t}^{i}, \\
m_{n z}^{e} & =m_{n z}^{i}, \\
u_{n}^{e}-u_{n}^{i} & =\lambda_{n} \sigma_{n n}^{e}, \\
u_{t}^{e}-u_{t}^{i} & =\lambda_{t} \sigma_{n t}^{e}, \\
\phi^{e}-\phi^{i} & =\lambda_{\phi} m_{n z}^{e},
\end{aligned}
$$

where $\lambda_{n}, \lambda_{t}, \lambda_{z}$ are the interface parameters Videla and Atroshchenko. The first three equations are equivalent to the condition $\boldsymbol{t}^{e}=\boldsymbol{t}^{i}$, while the last three equations we rewrite 


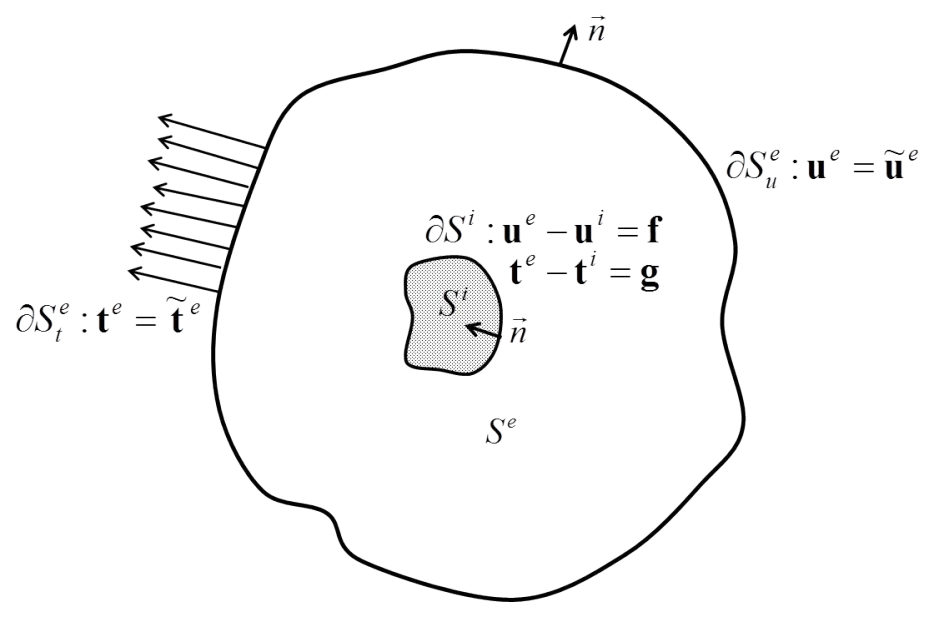

Figure 1: Inclusion boundary value problem. (7)

as

$$
\boldsymbol{u}^{e}-\boldsymbol{u}^{i}=\boldsymbol{A}(\boldsymbol{x}) \boldsymbol{t}^{e}
$$

where

$$
\boldsymbol{A}(\boldsymbol{x})=\left(\begin{array}{ccc}
n_{1}^{2} \lambda_{n}+n_{2}^{2} \lambda_{t} & n_{1} n_{2}\left(\lambda_{n}-\lambda_{t}\right) & 0 \\
n_{1} n_{2}\left(\lambda_{n}-\lambda_{t}\right) & n_{2}^{2} \lambda_{n}+n_{1}^{2} \lambda_{t} & 0 \\
0 & 0 & \lambda_{\phi}
\end{array}\right)
$$

Therefore, the second boundary value problem that we consider in this paper is written as

$$
\begin{aligned}
L^{i}\left(\partial_{x}\right) \boldsymbol{u}^{i} & =0 \text { in } S^{i}, \\
L^{e}\left(\partial_{x}\right) \boldsymbol{u}^{e} & =0 \text { in } S^{e} \\
\boldsymbol{u}^{e} & =\tilde{\boldsymbol{u}}^{e} \text { on } \partial S_{u}^{e}, \\
\boldsymbol{t}^{e} & =\tilde{\boldsymbol{t}}^{e} \text { on } \partial S_{t}^{e}, \\
\boldsymbol{u}^{e}-\boldsymbol{u}^{i} & =\boldsymbol{A}(\boldsymbol{x}) \boldsymbol{t}^{e} \text { on } \partial S^{i}, \\
\boldsymbol{t}^{e} & =\boldsymbol{t}^{i} \text { on } \partial S^{i} .
\end{aligned}
$$

\section{Boundary integral equations.}

The boundary integral equations of plane Cosserat elasticity for an arbitrary domain $S$ with boundary $\partial S$ and outward normal $\vec{n}$ are formulated as follows:

$$
\frac{1}{2} u_{i}(\boldsymbol{x})+f_{\partial S} P_{i j}(\boldsymbol{x}, \boldsymbol{y}) u_{j}(\boldsymbol{y}) d s_{y}-\int_{\partial S} D_{i j}(\boldsymbol{x}, \boldsymbol{y}) t_{j}(\boldsymbol{y}) d s_{y}=0, \quad i, j=1,2,3 .
$$

where $\boldsymbol{x} \in \partial S$ is called a source point, $\boldsymbol{y} \in \partial S$ is a field point. Matrices of fundamental solutions $D_{i j}(\boldsymbol{x}, \boldsymbol{y}), P_{i j}(\boldsymbol{x}, \boldsymbol{y})$ are given in Atroshchenko [a]. According to their asymptotic behaviour in the vicinity of $\boldsymbol{x}=\boldsymbol{y}$, which explained in details in Atroshchenko and Bordas [2015], components $D_{11}, D_{22}, D_{33}$ have logarithmic singularity, while $P_{12}$ and $P_{21}$ are singular and the corresponding integrals are understood in the sense of Cauchy Principal Value, as indicated by sign $f$.

In what follows $D_{i j}^{i}(\boldsymbol{x}, \boldsymbol{y}), P_{i j}^{i}(\boldsymbol{x}, \boldsymbol{y})$ denote matrices of fundamental solutions corresponding to the inclusion, described by material parameters with superscript $i$, while $D_{i j}^{e}(\boldsymbol{x}, \boldsymbol{y})$, 
$P_{i j}^{e}(\boldsymbol{x}, \boldsymbol{y})$ correspond to domain $S^{e}$. Equation $(12)$ can be directly prescribed for domain $S^{e}$ as

$$
\frac{1}{2} u_{i}^{e}(\boldsymbol{x})+f_{\partial S^{e} \cup \partial S^{i}} P_{i j}^{e}(\boldsymbol{x}, \boldsymbol{y}) u_{j}^{e}(\boldsymbol{y}) d s_{y}-\int_{\partial S^{e} \cup \partial S^{i}} D_{i j}^{e}(\boldsymbol{x}, \boldsymbol{y}) t_{j}^{e}(\boldsymbol{y}) d s_{y}=0
$$

For domain $S^{i}$ due to the inward orientation of the normal, the equation reads as follows:

$$
\frac{1}{2} u_{i}^{i}(\boldsymbol{x})-f_{\partial S^{i}} P_{i j}^{i}(\boldsymbol{x}, \boldsymbol{y}) u_{j}^{i}(\boldsymbol{y}) d s_{y}+\int_{\partial S^{i}} D_{i j}^{i}(\boldsymbol{x}, \boldsymbol{y}) t_{j}^{i}(\boldsymbol{y}) d s_{y}=0
$$

Applying the jump boundary conditions of (7) equation (14) can be re-written as:

$$
\frac{1}{2} u_{i}^{e}(\boldsymbol{x})-f_{\partial S^{i}} P_{i j}^{i}(\boldsymbol{x}, \boldsymbol{y}) u_{j}^{e}(\boldsymbol{y}) d s_{y}+\int_{\partial S^{i}} D_{i j}^{i}(\boldsymbol{x}, \boldsymbol{y}) t_{j}^{e}(\boldsymbol{y}) d s_{y}=p_{i}(\boldsymbol{x})
$$

where

$$
p_{i}(\boldsymbol{x})=\frac{1}{2} f_{i}(\boldsymbol{x})-f_{\partial S^{i}} P_{i j}^{i}(\boldsymbol{x}, \boldsymbol{y}) f_{j}(\boldsymbol{y}) d s_{y}+\int_{\partial S^{i}} D_{i j}^{i}(\boldsymbol{x}, \boldsymbol{y}) g_{j}(\boldsymbol{y}) d s_{y} .
$$

If boundary conditions (11) are used instead of (7), then eq. (14) becomes

$$
\begin{aligned}
\frac{1}{2} u_{i}^{e}(\boldsymbol{x}) & -f_{\partial S^{i}} P_{i j}^{i}(\boldsymbol{x}, \boldsymbol{y}) u_{j}^{e}(\boldsymbol{y}) d s_{y}+\int_{\partial S^{i}} D_{i j}^{i}(\boldsymbol{x}, \boldsymbol{y}) t_{j}^{e}(\boldsymbol{y}) d s_{y} \\
& -\frac{1}{2} A_{i j}(\boldsymbol{x}) t_{j}(\boldsymbol{x})+f_{\partial S^{i}} P_{i k}^{i}(\boldsymbol{x}, \boldsymbol{y}) A_{k j}(\boldsymbol{y}) t_{j}^{e}(\boldsymbol{y}) d s_{y}=0,
\end{aligned}
$$

Then the full system of boundary integral equations for the inclusion problem is given by $130+15$ or 13$)+17)$, where $u^{e}(\boldsymbol{x})$ and $t^{e}(\boldsymbol{x})$ are both unknown along the entire inclusion interface $\partial S^{i}$, while on the outer boundary $\partial S^{e}$ the equations are solved for $u^{e}(\boldsymbol{x})$ on $\partial S_{t}^{e}$ and for $t^{e}(\boldsymbol{x})$ on $\partial S_{u}^{e}$.

For the discretization of these BIEs we use a classical approach with quadratic Lagrange basis functions. For the evaluation of all weakly-singular integrals Telles transform Telles and Oliveira [1994] is used, while the singular integrals are evaluated using the singularity subtraction technique (SST), based on the asymptotic expansions of the matrices of fundamental solutions, given in Atroshchenko and Bordas [2015]. After the values of displacements/microrotation and tractions/couple tractions are evaluated along the boundary, the values of $\boldsymbol{u}^{i}, \boldsymbol{u}^{e}$ and $\boldsymbol{t}^{i}, \boldsymbol{t}^{e}$ inside the inclusion domain $S^{i}$ and the matrix domain $S^{e}$ respectively can be calculated using the micropolar analogues of Somiglina's displacement and stress identities, described in Atroshchenko and Bordas [2015]. The expressions for two more matrices of fundamental solutions, used in Somiglina's representation of the stresses and couple stresses inside a domain, are provided in a ready-to-use form in Atroshchenko a.

\section{Numerical results}

\subsection{Example 1. Circular inclusion in an infinite domain under remote tension: perfect interface.}

In the first example we consider an infinite plate subjected to the uniform tension and containing a circular inclusion with perfect interface, as shown in Fig.2. To take the full advantage of the boundary element method for problems with infinite domains, we seek 
the solution of this problem as a superposition of two solutions (Fig.3). The first one corresponds to the problem of an infinite plate in tension, without the inclusion, which is given in Paul and Sridharan [1981] as:

$$
u_{1}^{e}=\frac{\sigma_{0}}{2 G^{e}}\left(1-\nu^{e} x_{1}\right), \quad u_{2}^{e}=-\frac{\sigma_{0}}{2 G^{e}}\left(\nu^{e} x_{2}\right), \quad u_{3}^{e}=\phi_{3}^{e}=0 .
$$

The second solution corresponds to the problem of an inclusion in the infinite domain

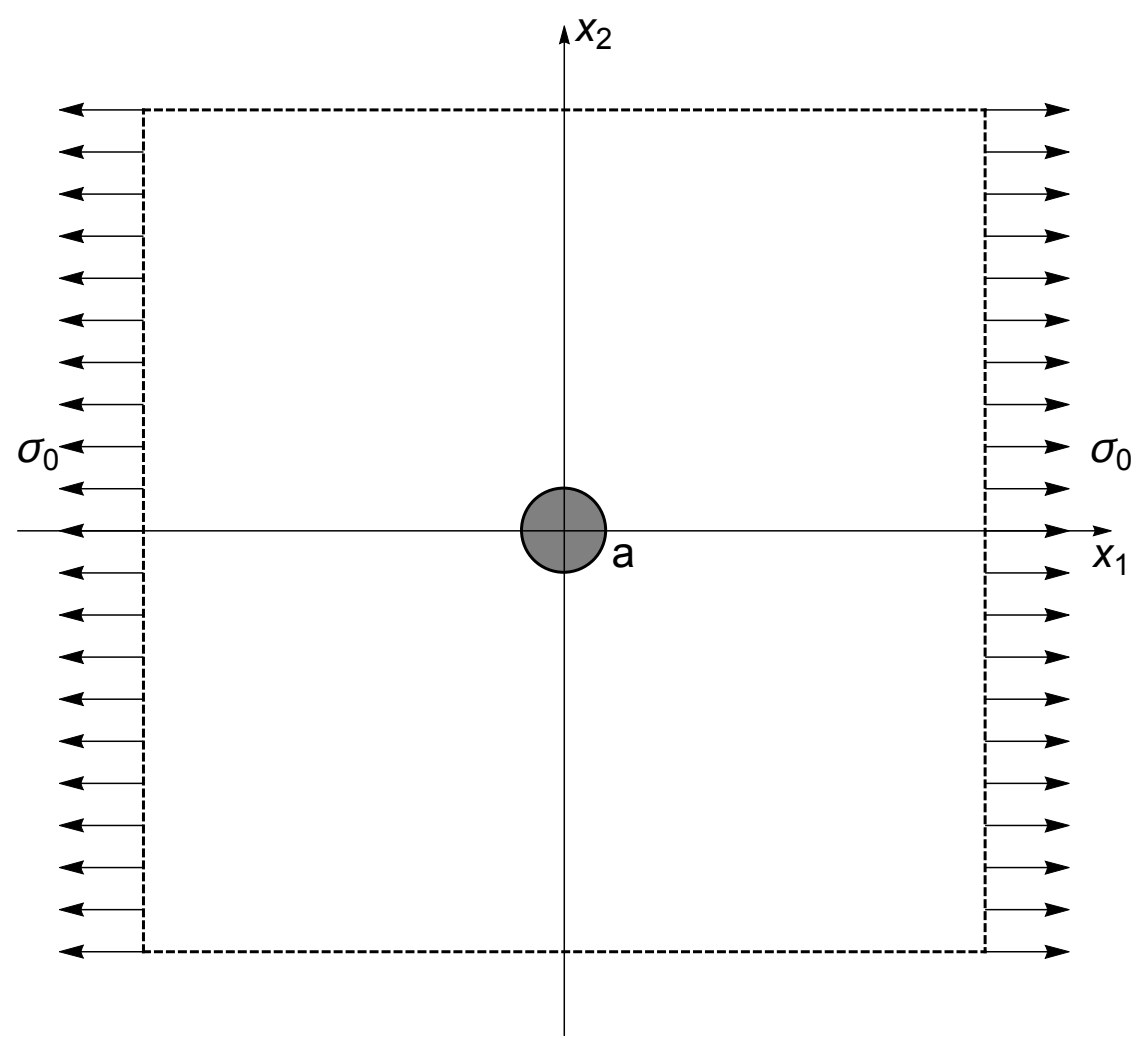

Figure 2: Circular inclusion in an infinite plate under remote tension.

with displacements/microrotations and stresses/couple stresses vanishing at infinity, while on the boundary of the inclusion

$$
\begin{aligned}
& u_{1}^{e}-u_{1}^{i}=f_{1}=-\frac{\sigma_{0}}{2 G^{e}}\left(1-\nu^{e} x_{1}\right), \\
& u_{2}^{e}-u_{2}^{i}=f_{2}=\frac{\sigma_{0}}{2 G^{e}}\left(\nu^{e} x_{2}\right) \\
& u_{3}^{e}-u_{3}^{i}=f_{3}=0
\end{aligned}
$$

and jump in tractions is given as:

$$
\begin{aligned}
& t_{1}^{e}-t_{1}^{i}=g_{1}=-\sigma_{0} n_{1} \\
& t_{2}^{e}-t_{2}^{i}=g_{2}=0 \\
& t_{3}^{e}-t_{3}^{i}=g_{3}=0
\end{aligned}
$$

Therefore, for the boundary element modelling we only consider the circular boundary $\partial S^{i}$ with inward normal $\vec{n}$ and functions $\boldsymbol{f}$ and $\boldsymbol{g}$ given by eq.(19), (20).

The analytical solution for this problem and the detailed study of the dependence of stress concentration on the material parameters is given in Videla and Atroshchenko, 


\begin{tabular}{|c|c|c|c|c|}
\hline & $\ell^{i} / a$ & $N_{i}$ & $\ell^{e} / a$ & $N_{e}$ \\
1 & 0.001 & 0.001 & 0.001 & 0.001 \\
2 & 0.001 & 0.001 & 1.000 & 0.900 \\
3 & 1.000 & 0.900 & 0.001 & 0.001 \\
4 & 0.750 & 0.500 & 0.750 & 0.750 \\
5 & 0.100 & 0.750 & 0.750 & 0.750 \\
6 & 0.500 & 0.900 & 1.000 & 0.750 \\
7 & 0.500 & 1.000 & 2.000 & 1.000 \\
\hline
\end{tabular}

Table 1: Material parameters for the study cases in example 1.

Atroshchenko [b]. The finite element method used as a further verification point was implemented using the DOLFIN Logg and Wells 2010 finite element library using standard quadratic Lagrangian elements for the displacements and linear Lagrangian elements for the microrotations. We used a graded triangular mesh generated using gmsh Geuzaine and Remacle [2009]. The code to generate all of the finite element results in this paper, is available at ?. Note that this standard displacement finite element formulation can not handle the couple-stress limiting case when $N=1$ due to a numerical locking effect. To mimic the effect of an infinite domain, we use a mesh with sides of length 20a. We use symmetry boundary conditions so that we only have to model one-quarter of the plate. Here we show the numerical results for 14 cases of the material parameters given in table 1 and $g=G^{i} / G^{e}=0.5, g=2$. In all cases the remaining material parameters were fixed at $\nu^{i}=0.25, \nu^{e}=1 / 3$. Note, that case 1 corresponds to the solution for classical elasticity, case 2 represents the inclusion, described by classical elasticity, in a strongly micropolar matrix, case 3 represents a strongly micropolar inclusion in a matrix, described by classical elasticity, case 4 corresponds to equal characteristic lengths, case 5 to equal couple numbers, case 6 represents a general variation of all material parameters, and case 7 corresponds to the limit case of couple-stress elasticity. In case 7, the results obtained in the present work are in an excellent agreement with the data from Weitsman 1965].

The stress concentration factor (SCF) is defined as

$$
\mathrm{SCF}=\max \left\{\frac{\sigma_{\theta \theta}^{e}(\pi / 2)}{\sigma_{0}}, \frac{\sigma_{\theta \theta}^{i}(\pi / 2)}{\sigma_{0}}\right\}
$$

The results in terms of the SCFs for all study cases (1) are given in Table 2 for $g=0.5$ and in Table 3 for $g=2$, where the excellent agreement between the analytical solution, BEM and FEM results is shown. In all cases, the BEM results were obtained by discretizing the inclusion contour with 36 elements, gradually refined towards the points $\theta= \pm \pi / 2$. The FEM results are significantly less accurate than the BEM results, despite the significantly higher number of degrees of freedom required. This finding adds further confirmation to the already well-known superiority of BEM in accurate resolution of stress concentrations around cracks and inclusions in standard elasticity.

\subsection{Example 2. Circular inclusion in an infinite domain under remote tension: homogeneously imperfect interface.}

As a second example, we consider a problem of a circular inclusion in an infinite plate subjected to a uni-axial tension. The interface between the inclusion and the matrix is assumed to be homogeneously imperfect, i.e. described by eq. (11) with three interface 

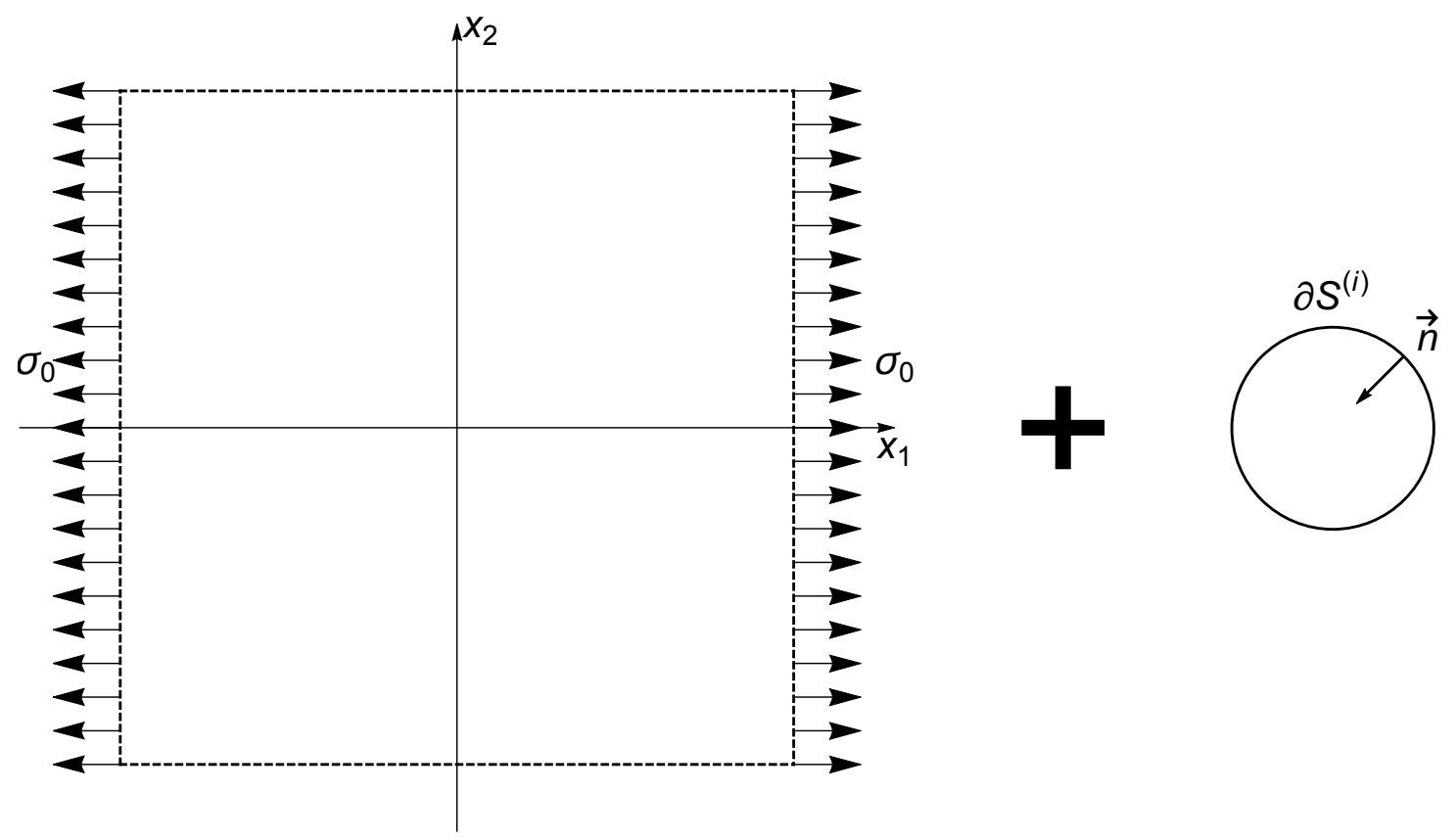

Figure 3: Superposition of the solutions for the inclusion problem.

\begin{tabular}{|l|c|c|c|c|}
\hline & Analytical solution & BEM & FEM & error BEM \\
1 & 1.57576 & 1.57540 & 1.57552 & $2 \times 10^{-4}$ \\
2 & 1.42722 & 1.42705 & 1.42797 & $1 \times 10^{-4}$ \\
3 & 1.57576 & 1.57550 & 1.57551 & $2 \times 10^{-4}$ \\
4 & 1.46478 & 1.46455 & 1.46529 & $2 \times 10^{-4}$ \\
5 & 1.46707 & 1.46689 & 1.46756 & $1 \times 10^{-4}$ \\
6 & 1.44876 & 1.44855 & 1.44926 & $1 \times 10^{-4}$ \\
7 & 1.35890 & 1.35871 & - & $2 \times 10^{-4}$ \\
\hline
\end{tabular}

Table 2: SCF given by $\sigma_{\theta \theta}^{e}(\pi / 2)$ for $g=0.5$.

parameters $\lambda_{n}, \lambda_{t}, \lambda_{\phi}$. This problem was studied analytically in Videla and Atroshchenko. In the present work we demonstrate the application of the boundary element method for the particular cases of the material and interface parameters, i.e. study case 6 of (1), for $g=0.5,2$ the following values of the interface parameters given in table 4 The boundary conditions (11) are further superimposed with the solution 19, 20) for an infinite plate without a hole, which allows to only consider the inclusion interface as a boundary for the BEM-discretization.

The results for both $\sigma_{\theta \theta}^{e}(\pi / 2) / \sigma_{0}$ and $\sigma_{\theta \theta}^{i}(\pi / 2) / \sigma_{0}$ are given in Table 5 . 


\begin{tabular}{|l|c|c|c|c|}
\hline & Analytical solution & BEM & FEM & error BEM \\
1 & 1.14872 & 1.14864 & 1.14731 & $7 \times 10^{-5}$ \\
2 & 1.27054 & 1.27086 & 1.26864 & $3 \times 10^{-4}$ \\
3 & 1.14872 & 1.14890 & 1.14729 & $2 \times 10^{-4}$ \\
4 & 1.24128 & 1.24134 & 1.23940 & $5 \times 10^{-5}$ \\
5 & 1.24914 & 1.24907 & 1.24673 & $6 \times 10^{-5}$ \\
6 & 1.26510 & 1.26486 & 1.26229 & $2 \times 10^{-4}$ \\
7 & 1.38507 & 1.38421 & - & $6 \times 10^{-4}$ \\
\hline
\end{tabular}

Table 3: SCF given by $\sigma_{\theta \theta}^{i}(\pi / 2)$ for $g=2$.

\begin{tabular}{|c|c|c|c|}
\hline & $\lambda_{n} G^{e}$ & $\lambda_{t} G^{e}$ & $\lambda_{\phi} G^{e}$ \\
$\mathrm{a}$ & 0.000 & 0.000 & 0.000 \\
$\mathrm{~b}$ & 10.00 & 10.00 & 10.00 \\
$\mathrm{c}$ & 10.00 & 0.000 & 0.000 \\
$\mathrm{~d}$ & 0.000 & 10.00 & 0.000 \\
$\mathrm{e}$ & 0.000 & 0.000 & 10.00 \\
$\mathrm{f}$ & 0.000 & 0.000 & 1000 \\
\hline
\end{tabular}

Table 4: Interface parameters for the study cases in example 2.

\begin{tabular}{|c|c|c||c|c|c|}
\hline & $\sigma_{\theta \theta}^{e}(\pi / 2) / \sigma_{0}$ & $\sigma_{\theta \theta}^{i}(\pi / 2) / \sigma_{0}$ & & $\sigma_{\theta \theta}^{e}(\pi / 2) / \sigma_{0}$ & $\sigma_{\theta \theta}^{i}(\pi / 2) / \sigma_{0}$ \\
\hline $\mathrm{a}$ & $1.44898(0.02 \%)$ & $0.64667(0.009 \%)$ \\
$\mathrm{b}$ & $2.20480(0.008 \%)$ & $0.06604(0.01 \%)$ & $\mathrm{a}$ & $0.65396(0.003 \%)$ & $1.26511(0.0008 \%)$ \\
$\mathrm{c}$ & $1.59208(0.02 \%)$ & $0.49783(0.008 \%)$ & $2.19958(0.002 \%)$ & $0.06960(0.0006 \%)$ \\
$\mathrm{d}$ & $1.79991(0.008 \%)$ & $0.36191(0.05 \%)$ & $\mathrm{c}$ & $0.90642(0.01 \%)$ & $1.04033(0.001 \%)$ \\
$\mathrm{e}$ & $1.45798(0.02 \%)$ & $0.64976(0.009 \%)$ & $\mathrm{d}$ & $1.43881(0.006 \%)$ & $0.58924(0.01 \%)$ \\
$\mathrm{f}$ & $1.45875(0.02 \%)$ & $0.65002(0.009 \%)$ & $\mathrm{e}$ & $0.64292(0.003 \%)$ & $1.23772(0.0008 \%)$ \\
$\mathrm{f}$ & $0.64257(0.003 \%)$ & $1.23685(<0.0001 \%)$ \\
\hline
\end{tabular}

$$
\text { a) } g=0.5 \quad \text { b) } g=2
$$

Table 5: Values of $\sigma_{\theta \theta}^{e}(\pi / 2) / \sigma_{0}, \sigma_{\theta \theta}^{i}(\pi / 2) / \sigma_{0}$ for $g=0.5, g=2.0$ and various values of interface parameters. The numbers in parentheses is the error in comparison with the analytical solution.

The results in Table 5 were obtained with 68 elements (612 DOFs) for the discretization of the circle. In all cases a good agreement with the analytical solutions is achieved, with the error within $0.05 \%$.

As it can be observed in Table 5 for $g=0.5$ the maximum stress at point $\theta=\pi / 2$ is always observed in the matrix, while for $g=2.0$ the maximum stress is observed either in the inclusion, or in the matrix, depending on the values of the interface parameters. Note, that some values of the interface parameters lead to almost no stress concentration (case c) at point $\theta=\pi / 2$.

In fig. 4, 5 the distribution of $\sigma_{\theta \theta}(r) / \sigma_{0}$ for various values of the interface parameters is plotted along $\theta=\pi / 2$, which demonstrate a good agreement between the BEM-results and the analytical solution. It can be seen, that the stress distribution is much more dependent on the parameters $\lambda_{n}$ and $\lambda_{t}$ (cases b, c, d), characterizing bonds in the radial and circumferential directions, than on the parameter $\lambda_{\phi}$, characterizing jump in the microrotation. 
The detailed parametric study of this problem is done in Videla and Atroshchenko.

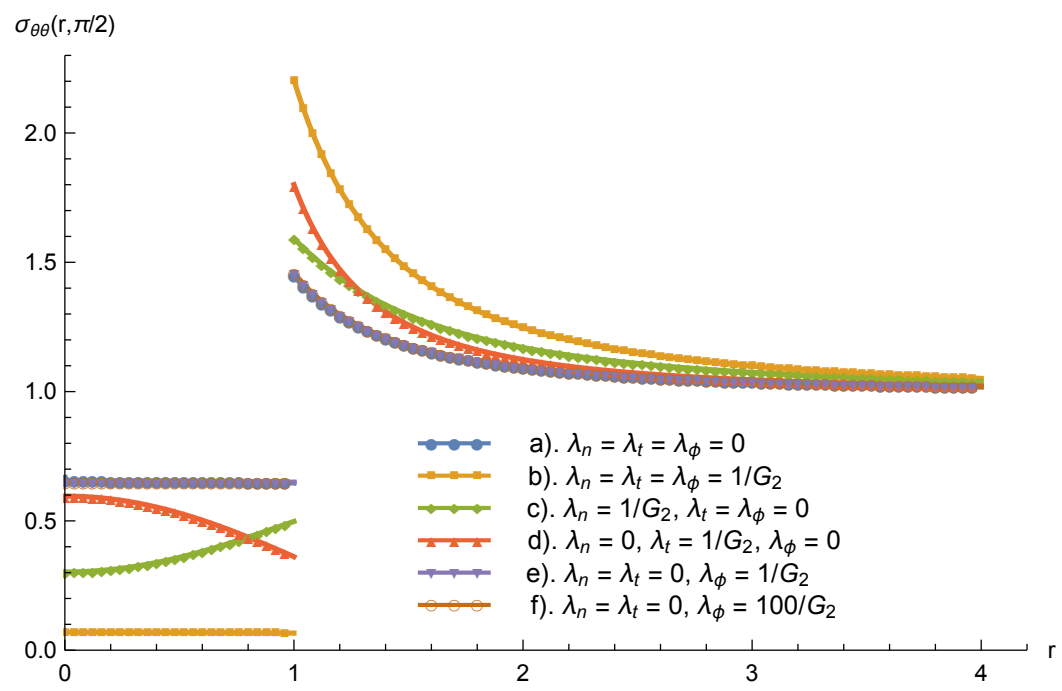

Figure 4: Distribution of $\sigma_{\theta \theta}(r, \pi / 2)$ for $g=0.5$ for different values of the interface parameters. BEM-data are shown by dots, while the corresponding analytical solutions are given by solid lines.

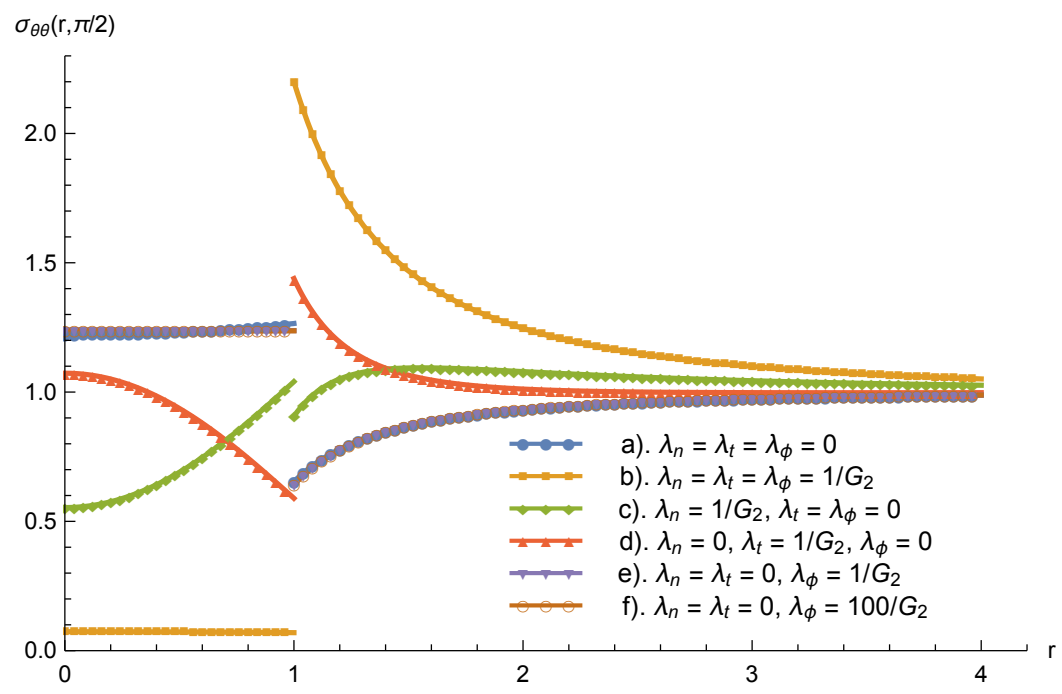

Figure 5: Distribution of $\sigma_{\theta \theta}(r, \pi / 2)$ for $g=2$ for different values of the interface parameters. BEM-data are shown by dots, while the corresponding analytical solutions are given by solid lines.

\subsection{Example 3. Circular inclusion with perfect interface in a finite plate under uni-axial tension.}

In the third example, we consider a circular inclusion with perfect interface in a finite plate of size $2 L \times 2 L$ subjected to the uniaxial tension, fig. 6 . We consider the same 14 study cases, as in the first example, but this time, we vary the ratio of $a / L$. The results in terms of the stress concentration factors are shown in tables. 6:7 in comparison with the finite element solution [bitbucketlink]. (mesh size, convergence study). The difference between 


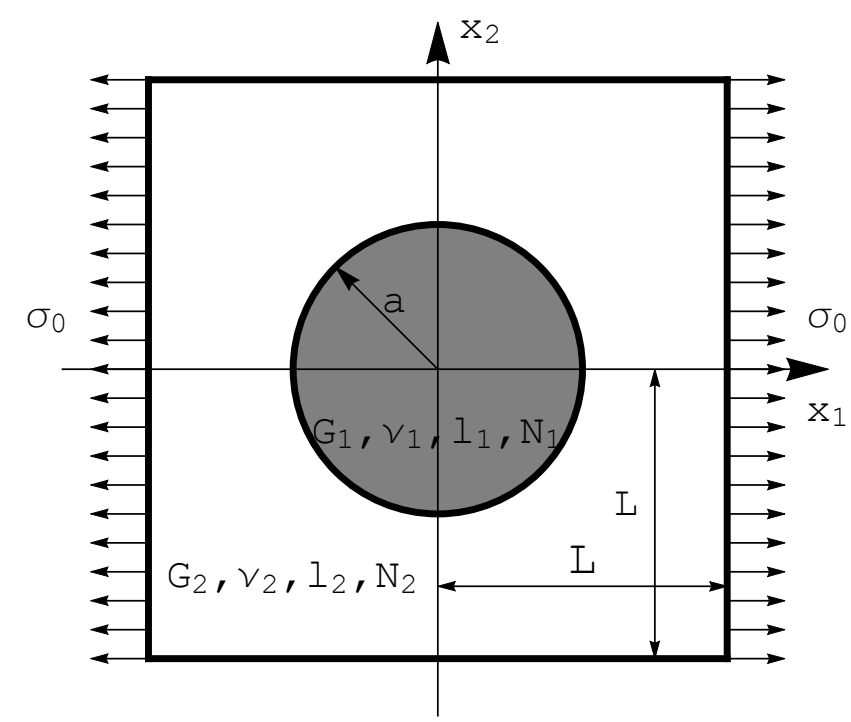

Figure 6: Circular inclusion in the finite plate.

two results is defined as

$$
\Delta=\frac{\mathrm{SCF}_{\mathrm{BEM}}-\mathrm{SCF}_{\mathrm{FEM}}}{\mathrm{SCF}_{\mathrm{FEM}}}
$$

It's interesting to note, that in the case of $g=2.0$ and $a / L=1.1$ no stress concentration occurs for some values of material parameters (cases 2,4 , and 7 ).

\subsection{Example 4. Inclusion of complex shape with perfect interface in a square plate.}

In the forth example, we consider an inclusion with perfect interface in a square plate of size $2 L \times 2 L$ subjected to the boundary conditions (fig,7):

$$
\begin{aligned}
& u_{1}=u_{2}=\phi=0 \text { at } x=-L, \\
& t_{1}=\sigma_{0}, t_{2}=t_{3}=0 \text { at } x=L .
\end{aligned}
$$

The size of the plate is set to $L=2 \mathrm{~mm}$ and the shape of the inclusion is given in the polar coordinates, associated with the point $(0,0)$ as

$$
r(\theta)=1+0.5 \sin (5(\theta+\pi / 4))(\mathrm{mm}) .
$$

Next, in order to illustrate the influence of the micropolar material constants on the deformation, we compare the numerical results for 8 sets of parameters:
a) $\ell^{i}=0.001 \mathrm{~mm}, N^{i}=0.001, \ell^{e}=0.001 \mathrm{~mm}, N^{e}=0.001$
b) $\ell^{i}=0.1 \mathrm{~mm}, N^{i}=0.25, \ell^{e}=0.5 \mathrm{~mm}, N^{e}=0.75$,
c) $\ell^{i}=0.9 \mathrm{~mm}, N^{i}=0.75, \ell^{e}=0.1 \mathrm{~mm}, N^{e}=0.9$,
d) $\ell^{i}=0.5 \mathrm{~mm}, N^{i}=1.0, \ell^{e}=0.75 \mathrm{~mm}, N^{e}=1.0$,

In each case $g=0.5,2.0$ and the remaining parameters were fixed to $\nu^{i}=0.25, \nu^{e}=1 / 3$. First, the (exaggerated) deformed contours are shown in Fig.8a, Fig.8b, where it can be seen that the influence of the chosen micropolar constants on deformation is slightly greater in the case of $g=0.5$. 


\begin{tabular}{|c|c|c|c|c|c|c|c|}
\hline & \multicolumn{4}{|c|}{$a / L=1.1$} & \multicolumn{3}{|c|}{$a / L=1.5$} \\
\hline study cases & BEM & FEM & \multicolumn{2}{|l|}{$\Delta$} & BEM & FEM & $\Delta$ \\
\hline 1. & 1.926 & 1.923 & \multicolumn{2}{|c|}{$2 \times 10^{-3}$} & 1.760 & 1.756 & $2 \times 10^{-3}$ \\
\hline 2. & 2.242 & 2.240 & \multicolumn{2}{|c|}{$9 \times 10^{-4}$} & 1.678 & 1.676 & $1 \times 10^{-3}$ \\
\hline 3. & 2.038 & 2.036 & \multicolumn{2}{|c|}{$1 \times 10^{-3}$} & 1.805 & 1.802 & $1 \times 10^{-3}$ \\
\hline 4. & 2.196 & 2.214 & \multicolumn{2}{|c|}{$2 \times 10^{-2}$} & 1.687 & 1.688 & $6 \times 10^{-4}$ \\
\hline 5. & 2.142 & 2.140 & \multicolumn{2}{|c|}{$1 \times 10^{-3}$} & 1.687 & 1.685 & $1 \times 10^{-3}$ \\
\hline 6. & 2.135 & 2.132 & \multicolumn{2}{|c|}{$9 \times 10^{-3}$} & 1.677 & 1.674 & $2 \times 10^{-3}$ \\
\hline 7. & 2.157 & - & \multicolumn{2}{|l|}{-} & 1.644 & - & - \\
\hline & & \multicolumn{4}{|c|}{$a / L=3.0$} & & \\
\hline & & $\overline{B E M}$ & FEM & \multicolumn{2}{|c|}{$\Delta$} & & \\
\hline & & 1.680 & 1.675 & \multicolumn{2}{|c|}{$3 \times 10^{-3}$} & & \\
\hline & & 1.478 & 1.476 & \multicolumn{2}{|c|}{$1 \times 10^{-3}$} & & \\
\hline & & 1.683 & 1.679 & \multicolumn{2}{|c|}{$2 \times 10^{-3}$} & & \\
\hline & & 1.522 & 1.521 & \multicolumn{2}{|c|}{$7 \times 10^{-4}$} & & \\
\hline & & 1.526 & 1.523 & \multicolumn{2}{|c|}{$2 \times 10^{-3}$} & & \\
\hline & & 1.502 & 1.498 & \multicolumn{2}{|c|}{$3 \times 10^{-3}$} & & \\
\hline & & 1.410 & - & \multicolumn{2}{|c|}{ - } & & \\
\hline
\end{tabular}

Table 6: SCF given by $\sigma_{\theta \theta}^{(e)}(\pi / 2)$ for $g=0.5$ and various ratios of $a / L$.

\begin{tabular}{|c|c|c|c|c|c|c|c|}
\hline & \multicolumn{4}{|c|}{$a / L=1.1$} & \multicolumn{3}{|c|}{$a / L=1.5$} \\
\hline study case & BEM & FEM & \multicolumn{2}{|l|}{$\Delta$} & BEM & FEM & $\Delta$ \\
\hline 1. & 1.107 & 1.106 & \multicolumn{2}{|c|}{$9 \times 10^{-4}$} & 1.149 & 1.148 & $9 \times 10^{-4}$ \\
\hline 2. & 0.952 & 0.953 & \multicolumn{2}{|c|}{$1 \times 10^{-3}$} & 1.138 & 1.138 & $1 \times 10^{-4}$ \\
\hline 3. & 1.048 & 1.048 & \multicolumn{2}{|c|}{$1 \times 10^{-4}$} & 1.089 & 1.089 & $1 \times 10^{-4}$ \\
\hline 4. & 0.976 & 0.962 & \multicolumn{2}{|c|}{$1 \times 10^{-2}$} & 1.144 & 1.139 & $4 \times 10^{-3}$ \\
\hline 5. & 1.037 & 1.037 & \multicolumn{2}{|c|}{$1 \times 10^{-4}$} & 1.166 & 1.164 & $2 \times 10^{-3}$ \\
\hline 6. & 1.012 & 1.012 & \multicolumn{2}{|c|}{$1 \times 10^{-4}$} & 1.160 & 1.159 & $9 \times 10^{-4}$ \\
\hline 7. & 0.993 & $\begin{array}{lll}- & - \\
\end{array}$ & \multicolumn{2}{|l|}{-} & 1.178 & - & - \\
\hline & & \multicolumn{4}{|c|}{$a / L=3.0$} & & \\
\hline & & BEM & FEM & \multicolumn{2}{|c|}{$\Delta$} & & \\
\hline & & 1.111 & 1.111 & \multicolumn{2}{|c|}{$1 \times 10^{-4}$} & & \\
\hline & & 1.237 & 1.236 & \multicolumn{2}{|c|}{$8 \times 10^{-4}$} & & \\
\hline & & 1.104 & 1.104 & \multicolumn{2}{|c|}{$1 \times 10^{-4}$} & & \\
\hline & & 1.209 & 1.203 & \multicolumn{2}{|c|}{$5 \times 10^{-3}$} & & \\
\hline & & 1.217 & 1.216 & \multicolumn{2}{|c|}{$8 \times 10^{-4}$} & & \\
\hline & & 1.235 & 1.234 & & & \\
\hline & & 1.342 & - & \multicolumn{2}{|c|}{$\begin{array}{c}8 \times 10^{-4} \\
-\end{array}$} & & \\
\hline
\end{tabular}

Table 7: SCF given by $\sigma_{\theta \theta}^{(i)}(\pi / 2)$ for $g=2.0$ and various ratios of $a / L$. 


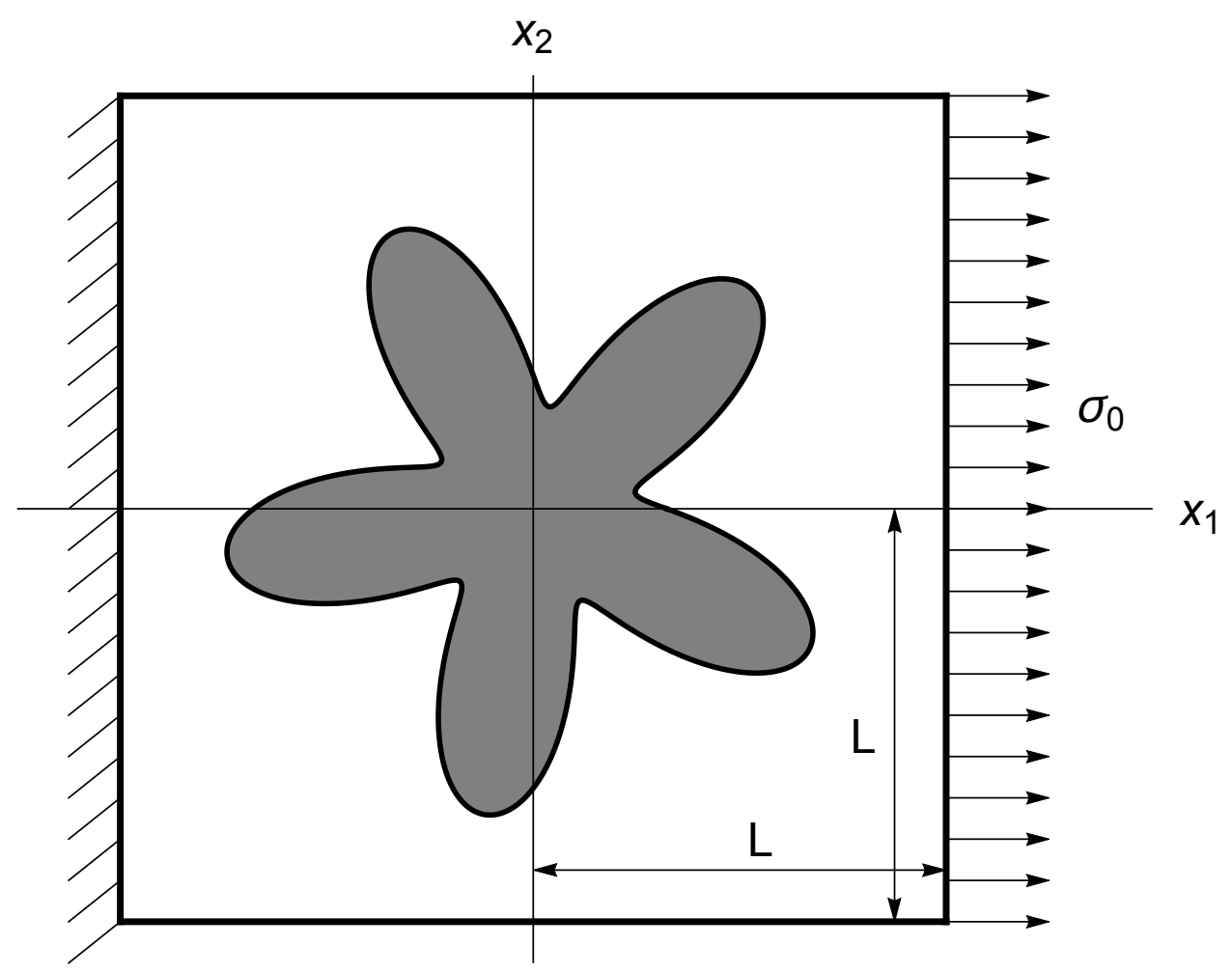

Figure 7: Inclusion of complex shape.

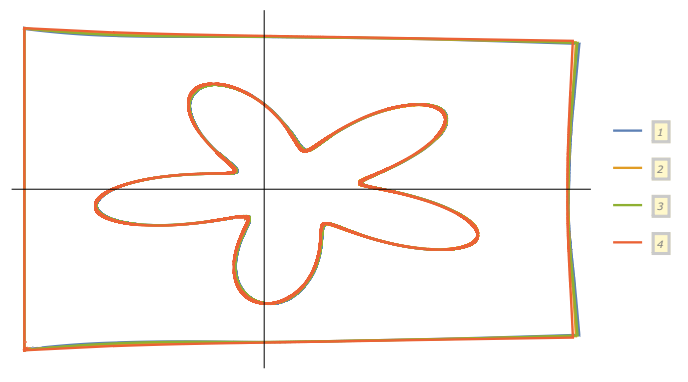

(a) $g=0.5$

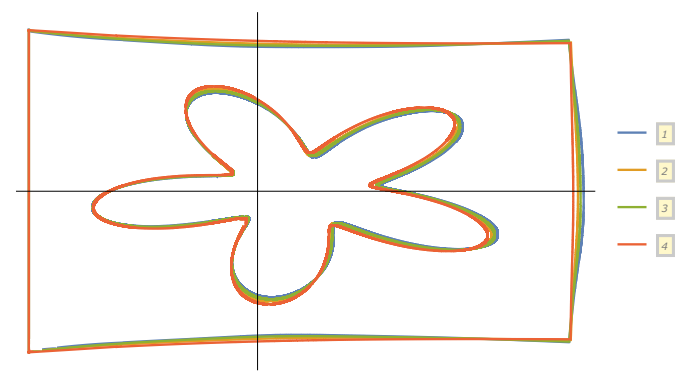

(b) $g=2.0$

Figure 8: Deformed contours for study cases 25 .

Next, the distribution of the normal and resultant stresses defined as

$$
\sigma_{n n}=t_{1} n_{1}+t_{2} n_{2}, \sigma_{\text {res }}=\left(t_{1}^{2}+t_{2}^{2}\right)^{1 / 2} .
$$

along the contour of the inclusion is shown in Fig 9a, Fig, 9b, Fig,10a, Fig.10b, where it can be seen that the micropolar effects on peak stresses (at positions $\theta_{1}, \ldots \theta_{6}$ ) are greater for stiffer inclusions. However, the detailed parametric study of interface failures is a subject of future studies.

\section{Conclusion}

In this paper the system of boundary integral equations for an inclusion problem in plane micropolar elasticity with imperfect interfaces was formulated and subsequently solved by the boundary element method. The boundary element method is shown to be an efficient tool for numerical analysis of boundary value problems with perfect and imperfect 


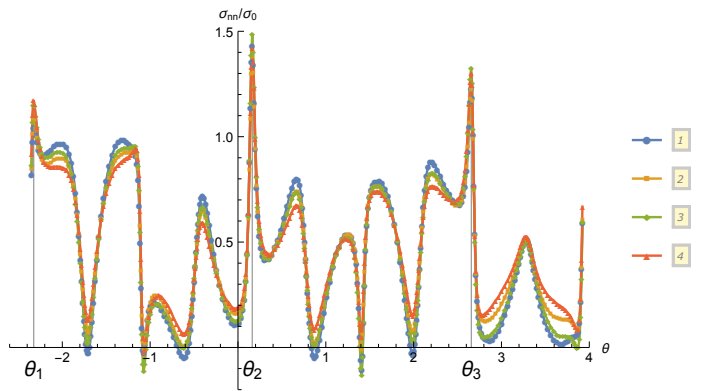

(a) $\sigma_{n n}$

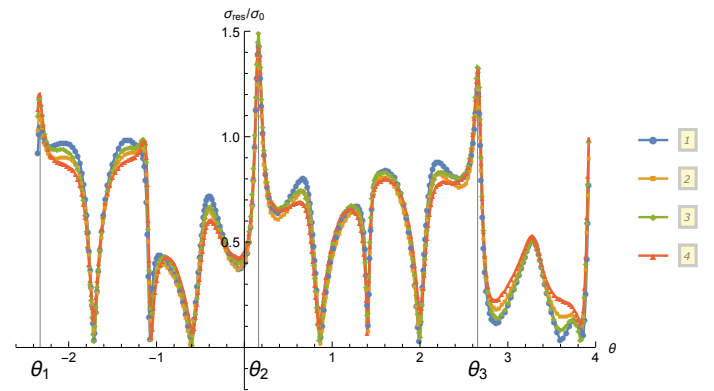

(b) $\sigma_{\text {res }}$

Figure 9: Distribution of the normal and resultant stresses along the inclusion interface for $g=0.5$ for study cases (25).

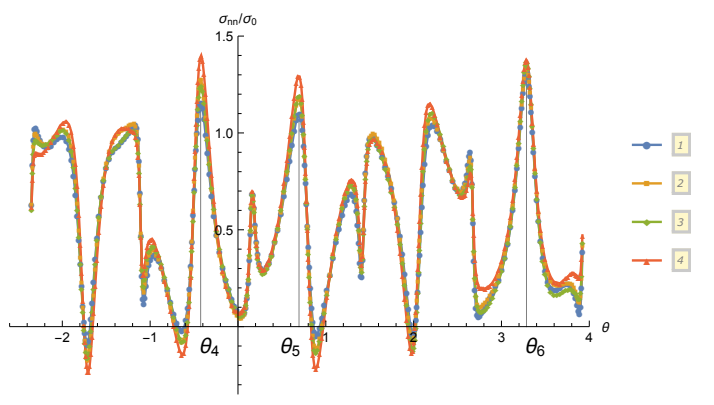

(a) $\sigma_{n n}$

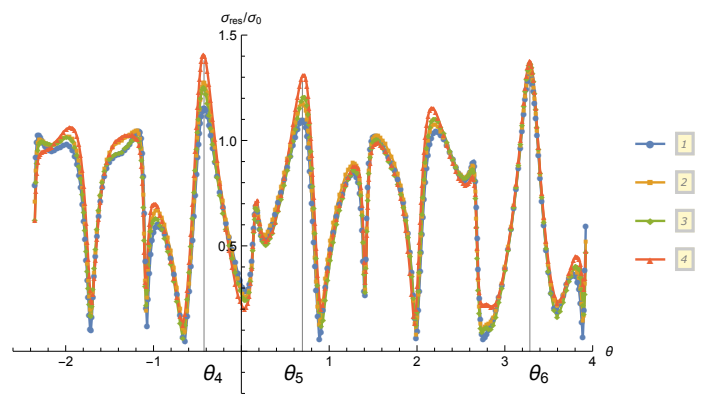

(b) $\sigma_{\text {res }}$

Figure 10: Distribution of the normal and resultant stresses along the inclusion interface for $g=2.0$ for study cases 25$]$.

interfaces. For a problem with imperfect interface it is shown that the jump conditions can be directly incorporated into the boundary integral equations.

The results presented in this paper can be further used for analysis of interface failure and crack heterogeneity interaction in Cosserat medium, which is the topic of ongoing work of our team. Another interesting research direction consists in establishing guaranteed homogenization bounds for Cosserat-heterogeneous materials using modern approaches based on stochastic mechanics such as Paladim et al.

\section{Supplementary material}

FEniCS code to produce the finite element method results can be found at Atroshchenko et al. 


\section{Acknowledgments}

Elena Atroshchenko and Javier Videla are partly supported by Fondecyt Chile, grant No 11130259 entitled "Boundary element modeling of crack propagation in micropolar materials".

Stéphane Bordas thanks funding for his time provided by the European Research Council Starting Independent Research Grant (ERC Stg grant agreement No. 279578) RealTCut Towards real time multiscale simulation of cutting in non-linear materials with applications to surgical simulation and computer guided surgery."

Stéphane Bordas is also grateful for the support of the Fonds National de la Recherche Luxembourg FWO-FNR grant INTER/FWO/15/10318764.

Jack Hale is partly supported by the National Research Fund, Luxembourg, and cofunded under the Marie Curie Actions of the European Commission (FP7-COFUND) Grant No. 6693582.

The authors also acknowledge the support of the Computational Science Research Priority at the University of Luxembourg for visits of Prof. Atroshchenko and to the University of Luxembourg for their support, in particular for access to the High Performance Computing cluster.

\section{References}

D. P. Adhikary and A. V. Dyskin. A Cosserat continuum model for layered materials. Computers and Geotechnics, 20(1):15-45, January 1997. ISSN 0266-352X. doi: 10.1016/ S0266-352X(96)00011-0. URL http://www.sciencedirect.com/science/article/ pii/S0266352X96000110.

Rashid K Abu Al-Rub. Continuum-based modeling of size effects in micro-and nanostructured materials. 2013.

Elena Atroshchenko. Plane Cosserat fundamental solutions, a. URL https:// sourceforge.net/projects/cosseratfundamentalsolutions/.

Elena Atroshchenko. Micropolar circular inclusion, b. URL https://sourceforge.net/ projects/micropolarinclusion/.

Elena Atroshchenko and Stéphane P. A. Bordas. Fundamental solutions and dual boundary element methods for fracture in plane Cosserat elasticity. Proc. R. Soc. A, 471(2179): 20150216, July 2015. ISSN 1364-5021, 1471-2946. doi: 10.1098/rspa.2015.0216. URL http://rspa.royalsocietypublishing.org/content/471/2179/20150216.

Elena Atroshchenko, Jack S. Hale, J. Videla, Stanislav Potapenko, and Stéphane P. A. Bordas. Micro-structured materials: inhomogeneities and imperfect interfaces in plane micropolar elasticity, a boundary element approach. supporting material. doi: 10.6084/ m9.figshare.4047462. URL https://dx.doi.org/10.6084/m9.figshare.4047462.

S. Bauer, M. Schfer, P. Grammenoudis, and Ch. Tsakmakis. Three-dimensional finite elements for large deformation micropolar elasticity. Computer Methods in Applied Mechanics and Engineering, 199(4144):2643-2654, October 2010. ISSN 0045-7825. doi: 10.1016/j.cma.2010.05.002. URL http://www.sciencedirect.com/science/article/ pii/S0045782510001441. 
A. J. Beveridge, M. A. Wheel, and D. H. Nash. The micropolar elastic behaviour of model macroscopically heterogeneous materials. International Journal of Solids and Structures, 50(1):246-255, January 2013. ISSN 0020-7683. doi: 10.1016/j.ijsolstr.2012.09.023. URL http://www.sciencedirect.com/science/article/pii/S0020768312004076.

D. Bigoni and W. J. Drugan. Analytical Derivation of Cosserat Moduli via Homogenization of Heterogeneous Elastic Materials. Journal of Applied Mechanics, 74(4):741-753, April 2006. ISSN 0021-8936. doi: 10.1115/1.2711225. URL http://dx.doi.org/10.1115/1. 2711225.

Huan Chen, Xiaoning Liu, Gengkai Hu, and Huang Yuan. Identification of material parameters of micropolar theory for composites by homogenization method. Computational Materials Science, 46(3):733-737, September 2009. ISSN 0927-0256. doi: 10.1016/ j.commatsci.2009.04.031. URL http://www.sciencedirect.com/science/article/ pii/S0927025609002055.

Eugne Cosserat and Franois Cosserat. Sur la thorie de l'lasticit. Premier mmoire. 1896. URL http://www .numdam.org/item?id=AFST_1896_1_10_3-4_I1_0.

Rémy Dendievel, Samuel Forest, and G Canova. An estimation of overall properties of heterogeneous cosserat materials. Le Journal de Physique IV, 8(PR8):Pr8-111, 1998.

H Dong, J Wang, and MB Rubin. Cosserat interphase models for elasticity with application to the interphase bonding a spherical inclusion to an infinite matrix. International Journal of Solids and Structures, 51(2):462-477, 2014.

H Dong, J Wang, and MB Rubin. A nonlinear cosserat interphase model for residual stresses in an inclusion and the interphase that bonds it to an infinite matrix. International Journal of Solids and Structures, 62:186-206, 2015.

HL Duan, J Wang, ZP Huang, and ZY Luo. Stress concentration tensors of inhomogeneities with interface effects. Mechanics of Materials, 37(7):723-736, 2005.

Victor A. Eremeyev, Leonid P Lebedev, and Holm Altenbach. Foundations of Micropolar Mechanics. SpringerBriefs in Applied Sciences and Technology. Springer Berlin Heidelberg, Berlin, Heidelberg, 2013. ISBN 978-3-642-28352-9 978-3-642-28353-6. URL http://link.springer.com/10.1007/978-3-642-28353-6.

A. Cemal Eringen. Linear theory of micropolar elasticity. 1965. URL http://oai.dtic. mil/oai/oai?verb=getRecord\&metadataPrefix=html\&identifier=AD0473723.

J. Fatemi, F. Van Keulen, and P. R. Onck. Generalized Continuum Theories: Application to Stress Analysis in Bone. Meccanica, 37(4-5):385-396. ISSN 0025-6455, 1572-9648. doi: 10.1023/A:1020839805384. URL http://link.springer.com/article/10.1023/ A: 1020839805384 .

Samuel Forest, Rémy Dendievel, and Gilles R Canova. Estimating the overall properties of heterogeneous cosserat materials. Modelling and Simulation in Materials Science and Engineering, 7(5):829, 1999.

Samuel Forest, Francis Pradel, and Karam Sab. Asymptotic analysis of heterogeneous cosserat media. International Journal of Solids and Structures, 38(26):4585-4608, 2001. 
Christophe Geuzaine and Jean-Franois Remacle. Gmsh: A 3-D finite element mesh generator with built-in pre- and post-processing facilities. International Journal for Numerical Methods in Engineering, 79(11):1309-1331, September 2009. ISSN 1097-0207. doi: 10.1002/nme.2579. URL http://onlinelibrary.wiley.com/doi/10.1002/nme.2579/ abstract.

SC Gupta. An inhomogeneity problem in couple stress theory. In Proceedings of the Indian Academy of Sciences-Section A, volume 84, pages 181-193. Springer, 1976.

A. R. Hadjesfandiari and G. F. Dargush. Boundary element formulation for plane problems in couple stress elasticity. International Journal for Numerical Methods in Engineering, 89(5):618-636, February 2012. ISSN 1097-0207. doi: 10.1002/nme.3256. URL http: //onlinelibrary.wiley.com/doi/10.1002/nme.3256/abstract.

Quan-Zhang Huang, Zhi-Gao Xu, Hong-Fu Qiang, Guang Wang, and Xiao-Ping Zheng. Boundary element method for solid materials with multiple types of inclusions. Acta Mechanica, 226(2):547-570, July 2014. ISSN 0001-5970, 1619-6937. doi: 10.1007/s00707-014-1186-1. URL http://link. springer.com/article/10.1007/ s00707-014-1186-1.

D. Iean. Existence theorems in the theory of micropolar elasticity. International Journal of Engineering Science, 8(9):777-791, September 1970. ISSN 0020-7225. doi: 10.1016/ 0020-7225(70)90004-2. URL http://www.sciencedirect.com/science/article/pii/ 0020722570900042 .

I Jasiuk and M Ostoja-Starzewski. Planar cosserat elasticity of materials with holes and intrusions. Applied Mechanics Reviews, 48(11S):S11-S18, 1995.

J. Jeong, H. Ramzani, I. Mnch, and P. Neff. A numerical study for linear isotropic Cosserat elasticity with conformally invariant curvature. ZAMM - Journal of Applied Mathematics and Mechanics / Zeitschrift fr Angewandte Mathematik und Mechanik, 89(7):552-569, July 2009. ISSN 1521-4001. doi: 10.1002/zamm.200800218. URL http://onlinelibrary.wiley.com/doi/10.1002/zamm.200800218/abstract.

Lei Li and Shuisheng Xie. Finite element method for linear micropolar elasticity and numerical study of some scale effects phenomena in MEMS. International Journal of Mechanical Sciences, 46(11):1571-1587, November 2004. ISSN 0020-7403. doi: 10.1016/ j.ijmecsci.2004.10.004. URL http://www.sciencedirect.com/science/article/pii/ S0020740304002590.

Y. J. Liu, N. Nishimura, Y. Otani, T. Takahashi, X. L. Chen, and H. Munakata. A Fast Boundary Element Method for the Analysis of Fiber-Reinforced Composites Based on a Rigid-Inclusion Model. Journal of Applied Mechanics, 72(1):115-128, February 2005. ISSN 0021-8936. doi: 10.1115/1.1825436. URL http://dx.doi.org/10.1115/1. 1825436 .

Anders Logg and Garth N. Wells. DOLFIN: Automated Finite Element Computing. ACM Trans. Math. Softw., 37(2):20:1-20:28, April 2010. ISSN 0098-3500. doi: 10.1145/1731022.1731030. URL http://doi .acm.org/10.1145/1731022.1731030.

VA Lubarda. Circular inclusions in anti-plane strain couple stress elasticity. International journal of solids and structures, 40(15):3827-3851, 2003. 
S. G. Mogilevskaya and S. L. Crouch. A Galerkin boundary integral method for multiple circular elastic inclusions with homogeneously imperfect interfaces. International Journal of Solids and Structures, 39(18):4723-4746, September 2002. ISSN 0020-7683. doi: 10.1016/S0020-7683(02)00374-8. URL http://www.sciencedirect.com/science/ article/pii/S0020768302003748.

Ed H Mühlhaus, J Wiley, NY Ch, and Roderic Lakes. Continuum models for materials with microstructure. 1995.

Hans-Bernd Mühlhaus. Continuum Models for Layered and Blocky Rock. In Analysis and Design Methods, pages 209-230. Elsevier, 1993. ISBN 978-0-08-040615-2. URL http://linkinghub.elsevier.com/retrieve/pii/B9780080406152500149.

N. I. Muskhelishvili. Some Basic Problems of the Mathematical Theory of Elasticity. Springer Netherlands, Dordrecht, 1977. ISBN 978-90-481-8245-9 978-94-017-3034-1. URL http://link.springer.com/10.1007/978-94-017-3034-1.

John A Nairn. Numerical implementation of imperfect interfaces. Computational Materials Science, 40(4):525-536, 2007.

S Natarajan. On the application of the partition of unity method for nonlocal response of low-dimensional structures. Journal of Mechanical Behaviour of Materials, 23(5-6): 153-168, 2014.

S Natarajan, S Chakraborty, M Thangavel, S Bordas, and T Rabczuk. Size-dependent free flexural vibration behavior of functionally graded nanoplates. Computational Materials Science, 65(http://arxiv.org/abs/1203.3643):74-80, 2012.

S Natarajan, Pierre Kerfriden, D Roy Mahapatra, and Stéphane Pierre Alain Bordas. Numerical analysis of the inclusion-crack interaction by the extended finite element method. International Journal for Computational Methods in Engineering Science and Mechanics, 15(1):26-32, 2014.

Witold Nowacki. Theory of Asymmetric Elasticity. Elsevier Science \& Technology, 1986. ISBN 978-0-08-027584-0. Google-Books-ID: yfVQAAAAMAAJ.

Patrick R. Onck. Cosserat modeling of cellular solids. Comptes Rendus Mcanique, 330(11): 717-722, November 2002. ISSN 1631-0721. doi: 10.1016/S1631-0721(02)01529-2. URL http://www.sciencedirect.com/science/article/pii/S1631072102015292.

Marco Paggi and Peter Wriggers. Stiffness and strength of hierarchical polycrystalline materials with imperfect interfaces. Journal of the Mechanics and Physics of Solids, 60 (4):557-572, 2012.

Daniel-Alves Paladim, José Paulo Baptista de Almeida, Stéphane Bordas, and Pierre Kerfriden. Guaranteed error bounds in homogenisation: an optimum stochastic approach to preserve the numerical separation of scales. Computer Methods in Applied Mechanics 86 Engineering.

H. C. Park and R. S. Lakes. Cosserat micromechanics of human bone: Strain redistribution by a hydration sensitive constituent. Journal of Biomechanics, 19(5): 385-397, January 1986. ISSN 0021-9290. doi: 10.1016/0021-9290(86)90015-1. URL http://www.sciencedirect.com/science/article/pii/0021929086900151. 
H. S. Paul and K. Sridharan. The problem of a griffith crack in micropolar elasticity. International Journal of Engineering Science, 19(4):563-579, January 1981. ISSN 0020-7225. doi: 10.1016/0020-7225(81)90090-2. URL http://www.sciencedirect.com/science/ article/pii/0020722581900902.

Hamidrza Ramzani, Amine El-Hraiech, Jena Jeong, and Claude-Laurent Benhamou. Size effect method application for modeling of human cancellous bone using geometrically exact Cosserat elasticity. Computer Methods in Applied Mechanics and Engineering, 237240:227-243, September 2012. ISSN 0045-7825. doi: 10.1016/j.cma.2012.05.002. URL http://www.sciencedirect.com/science/article/pii/S0045782512001508.

A. Riahi and J. H. Curran. Comparison of the Cosserat Continuum Approach with Finite Element Interface Models in a Simulation of Layered Materials. Scientia Iranica. Transaction A, Civil Engineering, 17(1):39, 2010. URL http://search .proquest.com/ openview/ef92598e51ee74aac73ce2d513ab4781/1?pq-origsite=gscholar.

Peter Schiavone. Integral equation methods in plane asymmetric elasticity. Journal of Elasticity, 43(1):31-43. ISSN 0374-3535, 1573-2681. doi: 10.1007/BF00042453. URL http://link.springer.com/article/10.1007/BF00042453.

Hossein Talebi, Mohammad Silani, Stéphane PA Bordas, Pierre Kerfriden, and Timon Rabczuk. A computational library for multiscale modeling of material failure. Computational Mechanics, 53(5):1047-1071, 2014.

C. Tekoglu and P. R. Onck. Size effects in the mechanical behavior of cellular materials. Journal of Materials Science, 40(22):5911-5917. ISSN 0022-2461, 1573-4803. doi: 10.1007/s10853-005-5042-5. URL http://link.springer.com/article/10.1007/ s10853-005-5042-5.

J. C. F. Telles and R. F. Oliveira. Third degree polynomial transformation for boundary element integrals: Further improvements. Engineering Analysis with Boundary Elements, 13(2):135-141, January 1994. ISSN 0955-7997. doi: 10.1016/0955-7997(94)90016-7. URL http://www.sciencedirect.com/science/article/pii/0955799794900167.

J. Videla and Elena Atroshchenko. Analytical study of a circular inhomogeneity with homogeneously imperfect interface in plane micropolar elasticity. ZAMM - Journal of Applied Mathematics and Mechanics / Zeitschrift fr Angewandte Mathematik und Mechanik, In Press. doi: 10.13140/RG.2.1.1689.5846.

Yechiel Weitsman. Couple-Stress Effects on Stress Concentration Around a Cylindrical Inclusion in a Field of Uniaxial Tension. Journal of Applied Mechanics, 32(2):424-428, June 1965. ISSN 0021-8936. doi: 10.1115/1.3625817. URL http://dx.doi.org/10. $1115 / 1.3625817$.

Liu Xue-Hui and Fazil Erdogan. The crack-inclusion interaction problem. Engineering fracture mechanics, 23(5):821-832, 1986.

J. F. C. Yang and Roderic S. Lakes. Experimental study of micropolar and couple stress elasticity in compact bone in bending. Journal of Biomechanics, 15(2): 91-98, January 1982. ISSN 0021-9290. doi: 10.1016/0021-9290(82)90040-9. URL http://www.sciencedirect.com/science/article/pii/0021929082900409 
Julien Yvonnet, Q-C He, and C Toulemonde. Numerical modelling of the effective conductivities of composites with arbitrarily shaped inclusions and highly conducting interface. Composites Science and Technology, 68(13):2818-2825, 2008a.

Julien Yvonnet, H Le Quang, and Q-C He. An xfem/level set approach to modelling surface/interface effects and to computing the size-dependent effective properties of nanocomposites. Computational Mechanics, 42(1):119-131, 2008b.

Xujun Zhao, Stéphane PA Bordas, and Jianmin Qu. A hybrid smoothed extended finite element/level set method for modeling equilibrium shapes of nano-inhomogeneities. Computational Mechanics, 52(6):1417-1428, 2013a.

Xujun Zhao, Ravindra Duddu, Stéphane PA Bordas, and Jianmin Qu. Effects of elastic strain energy and interfacial stress on the equilibrium morphology of misfit particles in heterogeneous solids. Journal of the Mechanics and Physics of Solids, 61(6):1433-1445, $2013 b$. 